\title{
El movimiento de formación de profesores en cursos a distancia: notas para una discusión
}

\author{
Ronaldo Nunes Linhares (Unit/SE) ${ }^{1}$ \\ Valéria Pinto Freire (Unit/SE) ${ }^{* *}$
}

\section{Resumen}

Las transformaciones ocurridas en el campo del conocimiento de la segunda mitad del siglo pasado se pueden entender como movimiento de la reconfiguración visceral del paradigma de la simplificación y del reduccionismo de la modernidad que implica procesos cognoscitivos, modelos culturales, de las comunicaciones prácticas, recursos tecnológicos, de los dispositivos educativos y de la interacción de los procesos. En el campo de la educación, este movimiento entiende al ciudadano como constructor de una acción reflexiva continua en su práctica diaria, a través de los registros y de las memorias de vida y de sus formas más diversas de análisis, enriqueciendo su "terminal personal" de las interacciones sociales necesarias para comprender las fuentes de organización de una sociedad: ideas culturales, creencia, símbolos y mitos, materiales impresos en su "cerebro-alcohólico" para las normas y decisiones. Esta comunicación estudia el movimiento como el registro y las prácticas de la formación de profesores en la Licenciatura a distancia de la Universidad de Tiradentes, analizando la construcción de la identidad del estudiante/profesor en la formación, las configuraciones y disociaciones posibles center/edges de la vida relacionadas con las mismas técnicas de la modalidad a distancia y su incorporación como instrumento de autoformación, del comentario y del análisis del profesor práctico, en un ejercicio reflexivo en cuanto a el cual haga posible su reencounter ser y como educador.

Palabras claves: Educación en la distancia. Formación de profesores. Movimiento.

Título: O movimento de formação de professores em cursos a distância: notas para discussão

\section{Resumo}

As transformações ocorridas no campo do conhecimento da segunda metade do século passado podem ser entendidas como movimento da reconfiguração visceral do paradigma da simplificação e do reducionismo da modernidade que implica em processos cognoscitivos, modelos culturais, das comunicações praticas, recursos tecnológicos, dos dispositivos educativos e da interação dos processos. No campo da educação, este movimento compreende um cidadão como construtor de uma ação reflexiva continua em sua prática diária, através dos registros e das memórias da vida e de suas formas maias diversas de análises, enriquecendo seu "terminal pessoal", das interações sociais necessárias para compreender as fontes de organização de uma sociedade: idéias culturais, crenças, símbolos e mitos, materiais impressos em seu "cérebro-alcoólico" para as normas e determinações. Esta comunicação estuda o monumento como o registro e as práticas da formação de professores de Licenciatura a distância da Universidade de Tiradentes analisando a construção da identidade do estudante/professor na formação, configurações e dissociações possíveis center/edges da vida relacionada com as mesmas técnicas da modalidade a distância e sua incorporação como

\footnotetext{
${ }^{1}$ Professor Doutor da Universidade de Tiradentes. E-mail: ronaldo_linhares@unit.br

*** E-mail:valeria_freire@unit.br
} 
instrumento de formação, do comentário e das analises do professor prático em um exercício reflexivo enquanto permite seu reencontro como sendo um educador.

Palavras-chave: Educação a distância. Formação de professores. Monumento.

Title: The movement of teacher training courses in the distance: notes for discussion

\begin{abstract}
The occurred transformations in the field of the knowledge from the second half of the last century can be understood as a movement of visceral reconfiguration of the simplification and reducionista paradigm of modernity involving cognitive processes, practical cultural, comunicacionais models, technological resources, educational devices of interaction and processes. In the field of the education, this movement understands the citizen as edificatory of a continuous reflexive action on daily practical its, through registers and memories of life and its more diverse forms of analysis, enriching its "Personal Terminal", of social interactions capable to understand the sources of organization of a society: cultural ideas, beliefs, symbols and myths, printed matters in its "brain-spirit" for the norms and determination. This communication studies the memorial as life register and practices of formation of professors in the Licenciatura in the Distance of the Tiradentes University, analyzing the construction of the identity of the pupil/professor in formation, the configurations and possible displacements center/edges related to the same techniques of the modality in the distance and its incorporation as instrument of autoformação, comment and analysis of the practical professor, in a reflective exercise that makes possible its reencounter as to be and as educator.
\end{abstract}

Keywords: Education in the distance. Formation of professors. Memorial.

\title{
1. Introducción.
}

Las transformaciones ocurridas en el campo del conocimiento de la segunda mitad del siglo pasado se pueden entender como movimiento de la reconfiguración visceral del paradigma del simplificador y del reducionista de la modernidad que implica procesos de los cognitivos, modelos culturales, de los comunicacionais prácticos, recursos tecnológicos y los dispositivos de la interacción.

La perspectiva del transitoriedade y del navigability constante, de que caracteriza a contemporáneo de la sociedad, es fruta de un proceso positivo del transistion y de la crisis de paradigmas. En esta dirección, autores como E. Morin (2000), afirma que vivimos un nuevo momento en la historia de la humanidad.

Para Morin vivimos en una sociedad que preguntan incesantemente sus determinismos económicos, social, políticos, cultural e histórico. Vivimos la complejidad como humano de la condición. La identidad compleja, de oposición a la edad de la exclusión/de la reducción. Según él, construimos un nuevo paradigma de la "necesidad de haber abierto nuevo, racional y crítico, reflexivas, autocríticas, las teorías convenientes automóvil-a remodelan, o iguales 
automóvil-a se revolucionen (...), tarea del epistemologia complejo" (MORIN \& LÊMOIGNE, 2000, p.220).

Ésta es la característica que mejor identifica la sociedad de estos primeros años del tercer milenio. Por lo tanto sus pasos de la comprensión para la revisión del papel de la educación y, en especial, de las formas o de la modalidad de aprender y de enseñar, mediado para las tecnologías de la información y de la comunicación (TIC). La acción educativa es una acción de la comunicación social y la política, por lo tanto, no sólo se educa para aprender contenido, pero para aprender en la vida, para aprender leer, entender el mundo y transformarlo. De Ambrosio define la educación como "estrategia adoptada para que las sociedades permitan que los individuos son creativos y alcancen el máximo de sus capacidades y que son, social, capaz cooperar con la siguiente en su parte ordinaria" (D’AMBRÓSIO, 1998, p.242).

Este concepto de la educación también es compartido por Freire, para quiénes es el papel básico de la educación liberar a los individuos de los conceptos de los mitos e (pago diario) que los obstaculizan para saber la realidad críticamente, y sabiéndola, son capaces transformarla. La educación entendida como la acción y política sociales, los nuevos espacios y las relaciones para aprender y para construir el conocimiento obliga a sociedad que cree las posibilidades donde la libertad crítica puede emerger como acción que transforma, estableciendo nuevas relaciones conceptuales con el TIC y los medias de la masa y de sus idiomas más diversas. Educar es, por lo tanto muy más grande una responsabilidad social de lo que aquélla percibida y dibujada hasta entonces por la sociedad y sus miembros.

Así, es básico que los seres humanos de las acciones, la libertad y la responsabilidad, son regalos en la base de los comunicacionais, las relaciones importantes para el equilibrio social. Estas acciones exigen cambios en los procesos de la construcción del conocimiento en especial a ésos dirigidos hacia la formación de profesores/de pupilas o de las pupilas/de los profesores. Pensando de esta relación, observamos que estos últimos años los procesos del institucional de la educación habían sido obligados para moverse los practican de la formación de los profesores, que deben irse de un fabricante de proceso de memorias para hacer un comunicante y una acción creativa.

Si la comunicación es la relación que si caracteriza para la co-participación de los ciudadanos en curso de construcción del conocimiento, la educación especial y en la educación medió para el TIC, pues es la educación en la distancia, se presenta como posibilidad verdadera donde esta relación se debe consolidar como acción crítica y creativa. 
En vista de la necesidad de si el apropiarse de las conquistas y de los avances del conocimiento científico y tecnológico, en especial de ésos relacionó el TIC, podemos afirmar eso, porque una sociedad dominada para la información y la comunicación, para saberla es también entender la mundo-imagen.

Uno demuestra, de ahora en adelante, un nuevo momento para la educación, en especial para la educación "que considera un proceso de la apropiación de los nuevos códigos de los comunicacionais que pueden desalienar la escuela, y dotarla con una visión crítica que contribuya de modo que las nuevas generaciones entiendan a sociedad donde viven"l.

El desafío más allá de saber los comunicacionais nuevos de las idiomas, los aparatos tecnológicos nuevos, que amplían las posibilidades de comunicación del hombre, modifica la producción y la consumición de productos simbólicos, en él impulsa para pensar que ellos la educación y la escuela pues el político del espacio de la reflexión en estas idiomas debe construir nuevo practica en la formación de los profesores que tienen el TIC como mediando en curso de construcción del conocimiento y formación de la identidad del educador.

Este artículo es el resultado de un esfuerzo de entender de la experiencia de la formación de los profesores desarrollados por la universidad de Tiradentes en la modalidad de la educación en la distancia que las nuevas posibilidades de practican el elemento formativo que consideran historias de vidas de profesores en la formación, registrado en monumentos, de instrumentos de la formación y evaluación de ellos usted practica a profesores, momento de la reflexión en la acción y en la acción. Se ancla por lo tanto en tres bases.

Primer entiende la complejidad como lecho para una reflexión del epistemológica en el universo, el mundo, la sociedad y el hombre, buscando con esta diversa mirada, para reconstruir un itinerario que reconozca cosas entre otros la naturaleza subjetiva del conocimiento y como se impregna todo de nuestras lecturas apropiadas del mundo, de una "impresión cultural" sin la vuelta, reintroduciendo el ciudadano en el conocimiento y el conocimiento en el ciudadano, el explicitando una relación del indissociável entre el ciudadano que sabe y el fenómeno/el objeto que desea explicar, entender, entender.

Esto consideraba ejercicio del epistemológico en algunas áreas de las tomas del conocimiento el movimiento de la generalidad en la particular y el particular en la generalidad como perpetuo venir ser yo continúa, en un esfuerzo contra la separación (Morin, 1998) a

\footnotetext{
${ }^{1}$ SILVEIRA, Maria Helena. Entrevista concedida entre o período de agosto a novembro de 2000.

${ }^{2}$ O Imprinting termo (Konrad Lorentz) que dar conta da marca incontornável imposta pelas primeiras experiências do jovem animal. (2002:29)
} 
favor de un sistêmica y de una visión del autopoietica del conocimiento (Maturana; 1997, 1989). Considera en segundo lugar la construcción del conocimiento como un ejercicio constante seleccionar, a hierarquizar, aceptar y rechazar las ideas y la información, en función de significações de los mitológicas y de proyecciones imaginarias, básicas para la construcción social de la realidad, del enraizamento del conocimiento en la sociedad y de la integración del conocimiento/de la sociedad, pero, sobre todos, del anillo del recursivo en el cual el conocimiento está produciendo/producto una realidad sociocultural que lleve a cabo una dimensión del cognitiva intrínseco como producto de bio-antropo-sociocultural interacciones y tenga en la educación un espacio social privilegiado donde sus principios, reglas e instrumentos se deben construir colectivamente.

E finalmente, entiende lo practica de registros conmemorativos como acción donde está al mismo tiempo sujetos y se opone el profesor/la pupila, y donde si él observa, él analiza su práctico y si sea el reencontra en cuanto a un uno práctico que él considera la vida como espacio de la formación. El monumento como instrumento reflexivo de practica y el aprender con y en el práctico. La reflexión implica en la inmersión concienzuda del hombre en el mundo de su experiencia, un mundo cargado de connotaciones, los valores simbólicos, los intercambios, las correspondencias afectivas, los intereses sociales y los políticos de las escenas (PÉREZ GÓMEZ, 1995).

\section{El monumento en los cursos de la formación de profesores en la distancia de la UNIT}

En su libro "método IV", Edgar Morin, describe el conocimiento como producto de bio-antropo-sociocultural interacciones. Quizás comienza durante el período embrionario y eso tiene en la educación el espacio para la construcción de los principios, de las reglas y de los instrumentos del conocimiento. Este proceso de la construcción del conocimiento es un ejercicio constante para seleccionar, a hierarquizar, para aceptar y para rechazar las ideas y la información, en función de significações de los mitológicas y de proyecciones imaginarias, básicas para la construcción social de la realidad, del enraizamento del conocimiento en la sociedad y de la integración del conocimiento/de la sociedad, pero, sobre todos, del anillo del recursivo en el cual el conocimiento está produciendo/producto de una realidad sociocultural que lleve a cabo una dimensión del cognitiva intrínseco. 
Se consideran estos razonables estimada cuando están observada en el modelo real de la educación establecido de modernidad. Con respecto a la educación en la distancia el proceso de la construcción del conocimiento en una perspectiva general continúa ocurriendo de la misma forma, pero, si debe considerar la existencia de nuevos elementos por ejemplo: el proceso del mediatização/de la interacción, del papel de las tecnologías y de las idiomas de la comunicación y del concepto del sistema/de la red que redefine nuevos espacios y normas en las relaciones entre los ciudadanos. Considerando llama TIC pues los productos de la cultura total y de saber que los hombres y las mujeres de una cultura, para su manera del conocimiento, producen la cultura que produce su manera de saber, el dependiente de las condiciones sociocultural múltiples, que, en vuelta, condiciona y regeneró la cultura y que los individuos no son todos, y ni siempre, exactamente en las condiciones culturales más cerradas, máquinas triviales obedeciendo el impecablemente la orden social y las prescripciones culturales él es que si debe en la distancia considerar en un proceso de la educación las posibilidades de eso los individuos pueden ejercitar su capacidad de la autonomía de la comprensión de Morin en conocimiento/cultura de la relación/educación.

Para el absoluto, autor oficial, sacralizadas de los certezas: los mitos; el dogma, la ceguera, visión del alucinada, el arqui-determinismo de los paradigmas, la impresión de la normalización, el invariancia, la reproducción, toda que éste también implica, en la debilidad de los lugares de la impresión de las aberturas en la normalización, las líneas de desvío la evolución del conocimiento, las modificaciones en la reproducción estructura. Las progresiones corrosivas y la subversión de la duda: la existencia y el desarrollo, el brote de la súplica, la nueva mirada en fecha neutonio, de las revoluciones de los copernicanas, toda esto transforma las estructuras del pensamiento y los visiones del mundo.

Para utilizar a la ventaja que las posibilidades de modificación de la reproducción estructuran, siendo espacios construidos y/o los momentos donde las progresiones corrosivas y la subversión de la duda pueden contribuir para las transformaciones de las estructuras del pensamiento deben en la distancia ser uno de los objetivos de la adopción de técnicas y de instrumentos de aprender y de la educación en el uso de la metodología de la educación. En esta dirección estas metodologías deben privilegiar la construcción de la identidad profesional de los ciudadanos mientras que la reflexión activa en el proceso apropiado de la formación, de que debe ocurrir del hábito para colocar su acción y reacciones, sus impresiones en su crecimiento personal y profesional.

La contribución del avance tecnológico es un ejemplo. Los ambientes virtuales usados como espacios colectivos de aprender, si se han vuelto cada vez más hacia la creación de estas 
posibilidades. Las metodologías de Educación en la Distancia (EAD) buscan para ampliar a través de los mediaciones tecnológicos las posibilidades ilimitadas de interacción y el desarrollo de capacidades independientes de la reflexión en practica con la metodología de "casa", de los colaborativos proyecta, la producción colectiva de textos y de foros e incentivo la participación de discusiones virtuales en charlas. El Internet ha contribuido con los nuevos ambientes de la interacción y de la participación colectiva como el Blogs. De los personales hasta los temáticos, los blogs son ejemplos de los espacios construidos a convertirse publican a ciudadanos privados o los nuevos espacios públicos de la pelea del mí lo publico. En este campo de la formación del profesor, el monumento puede contribuir con este ejercicio constante para modificarse, con la subversión de dudas, las estructuras de la reproducción, construyendo espacios y/o los momentos pueden contribuir para transformar las estructuras del pensamiento. Pues resultó el elemento de las medidas formativas del somativa evaluasion no sólo, el efecto o los funcionamientos, pero llega a ser posibles observar y entender en metodológica de la manera, sistemático e individualizada el funcionamiento de cada pupila que hace así posible un ajuste que las ocasiones que aprenden amplíen, tiene como fuente de la preocupación la racionalidad objetiva del instrumento del alcance que da a énfasis aprender, la participación, los subjetividades, singularidades de los diversos ciudadanos en curso de adquisición del conocimiento.

El modelo de EAD considerado para la universidad de Tiradentes, porque la formación de profesores fueron desarrollados en convenio con los pasillos de ciudad del interior nordestal y tiene en la materia impresa material y el proceso de la tutela y del mediatização sus pilares básicos. Diferente de los procesos construidos de EAD que se mediarán para las tecnologías digitales y circundantes virtuales, los procesos que apoyan una oferta de la educación en la distancia basaron en materia impresa material, sin embargo mediado para los modelos de la tutela muy al lado del papel del profesor como sabemos, muy exija un esfuerzo más grande en crear los instrumentos y las condiciones de automóvilestudian que permiten en la pupila el ejercicio de la acción-reflexión-acción.

\section{De ese monumento hablamos.}

La expresión conmemorativa viene del conmemorativo latino y significa una escritura donde alguien dice hechos memorables. Para expresar emociones, las sensaciones, deseos, puntos de vista, dudas, certezas, fragiles, de presentar crítico, reflexiones, toda esto 
constituyen la pieza del monumento. Podemos también considerar el monumento como área de pertenecer del ciudadano/del objeto en la construcción de sus práxis didáctico-pedagógicos y diariamente de aprender dónde si establece una esfera de los intercambios interactivos eficaces y si espera eficiente.

Se articula exactamente a otros modelos más formales de la evaluación el monumento sin embargo que viene sirviendo para evaluar solamente aprender un instrumento que se debe interior levantar al grado más alto de significación de los práxis didáctico-pedagógicos de cada remitente/profesor como instrumento de la automóvil-evaluación, como canal de la interacción, dialogia donde la comunicación hace posible la construcción de un discurso del diario del receptor/de la pupila de donde las tranvías legítimas emergen que distinguen las relaciones de la bio-antropo-sociocultural, el rencor de las delimitaciones de la naturaleza de la educación para no reconocer en el discurso de la pupila su creencia, sus mitos, sus valores, sus emociones, sus incertidumbres, su señal de socorro e sensaciones. En esta dirección el monumento es el alambre que conduce de la interacción bidireccional que de tal manera logra el mensurasión del proceso de enseñar-aprender para el profesor de cuánto para la pupila, exactamente siendo reconocido el inviabilidad si en la distancia estableciendo en la educación una comunicación fluida y habitual.

La historia de la educación en la distancia en el Brasil, mediada para la materia impresa material viene demostrando que el uso del monumento ha contribuido en la una formación "habitus" de la reflexión en la acción, principalmente en el campo de la formación de profesores, convirtiéndose en un instrumento básico para la construcción de su identidad profesional mientras que de la educación.

Sabido principalmente en el mundo académico y de los cursos reales, el monumento es ancho del instrumento un ya usado en la elección de los profesores para la universidad, para certificar una silla o como opción para alguno en el trabajo académico. Sin embargo, la primera experiencia del uso del monumento en la formación cursa en la distancia ocurrió al comienzo del siglo con el curso para la formación de los profesores puestos _ PROFORMAÇÃO, producida para el Ministerio de la Educación (MEC) a través de la secretaría de la educación en la distancia. En la primera guía general del PROFORMAÇÂO, producida para el profesor Maria Antonieta. Cruz, $(2000 ; 31)$ y publicada en el período de lanzar del programa en el 2000, cuando presenta la metodología del curso, monumento fue descrita solamente como "documento escrito elaborado para el profesor de Cursista, dirigido para la guía de estudios y del llevada a través de profesor particular (...) a través del módulo". Dos años más adelante la misma guía, en una edición $3^{a}$ agregó que "en este 
documento, el profesor refleja en su paso en el curso, avances, dificultades, experiencias de pedagógico práctico y resultado”.

Esta misma metodología sirvió de base a los rastros del proyecto, de un programa de la formación de profesores en el nivel superior desarrollado por el gobierno de Minas Gerais con sociedad con todas las instituciones de la educación superior del estado y de la validez del credenciou de la oferta él de modo que fuera introducida como metodología de la evaluación del obligator del proyecto ofrecido de Licenciaturas en la modalidad en la distancia para la Universidade de Tiradentes (UNIT).

El monumento fue implantado inicialmente en el curso portugués de letras, con las pupilas/los profesores, que ejercieron ya en su mayoría lo practican pedagógico en escuelas municipales, con los grupos del pre-entrenamiento, del alfabetização y de la primera fase de la educación básica. A los actuales objetivos ya previamente, uno agregado más uno dirigió la construcción del texto escrito, puesto que la opción teórica del proyecto de letras fue dirigida la construcción de la lengua como elemento de la cultura que separaba sí mismo desde el concepto de la norma cultivada y de la lengua mientras que producto de la gramática normativa. Si por otra parte esta opción amplió la visión de la pupila en la construcción del texto, para otro, lo colocó en dificultades, principalmente éstos de cursos en la distancia delante de la organización clásica del texto y de su comumentimente normativo de la estructura cargado en la escuela.

Exactamente con experiencia en la sala de clase - más el de $40 \%$ de las pupilas/de los profesores tenía más de cinco años en sala de clase - el nivel los mismos en este campo estaba muy en este lado de esperado para los profesores que actuaban en curso de alfabetizasión de niños. Así el monumento pensó inicialmente para una producción cada bimaster, delante de esta realidad, semanario pasado para ser cargado.

Un documento subvenciona y dirige a profesores particulares y a pupilas, con los criterios de la evaluación que tuvieron que dirigir los pasados en la construcción y los registros de las ideas y primeras en la puntuación del monumento. Exactamente con este documento, habían sido muchos y distinguieron las dificultades iniciales hechas frente principalmente para las pupilas cuánto su capacidad de entender su crecimiento personal y las interrelaciones posibles entre el y la construcción o el refuerzo de la identidad profesional durante el proceso de aprendizaje. Dificultades relativas la estética, estructura y constitución del texto, el registro de los hechos y de las opiniones en el curso, la metodología y su nivel de la dedicación él; cuál las relaciones posibles entre el objeto del estudio práctico y el diarios, 
donde el curso contribuyó al avance y perfeccionar su práctico y reflejar en él pontuou la construcción de los primeros monumentos, de ese tenido sido entregan durante el primer semestre del curso.

\section{Lecho de monumento a proyecto de formación de profesor de Tiradentes Universidad.}

Interactivo relación que si establezca en los cursos dados en la modalidad de EAD no es ni más ni menos frágil de lo que existen los intercambios logrados en los cursos de la modalidad real, mucha sin embargo incorpora las modalidades que una diferencia significativa en qué dicen respecto a la época de la construcción de estimaba su. La preocupación de pedagógico práctico en la valuación y del dinamicidade de los procesos de los comunicacionais es muy reciente y en su uso actual como lecho del estruturante de enseñar-aprender de proceso.

$\mathrm{Al}$ explorar este sistema de implicaciones percibimos dos recortes de los exploratorios que consideremos ser capaces a clarear las condiciones generales donde podemos utilizar el monumento como metodología de la evaluación del obligator en los cursos del licenciatura en una distancia ofrecida por la Universidad de Tiradentes. El primer truncamiento dice respecto a la teoría de la comunicación formulada para Habermas (1998) centrado en un modelo de la interacción que nomine de racionalidad del comunicativa o de la acción del comunicativa donde los participantes buscan para alcanzar un acuerdo en la situación definitiva con la lengua o qué ella establece como "situación ideal de habla", justificado en cuatro niveles distintos de la validez, pero que se articulan entre sí mismo: cuál es dicho es inteligible, el contenido de cuál es dicho es verdad; el remitente si justifica para ciertas derechas sociales o las normas que se invocan en el uso de la lengua (respecto mutuo) el remitente son sinceras en lo que él dice, no intenta engañar ni persuadir el receptor según (autenticidad de los interlocutores) el autor cuando una de estas reglas se viola la comunicación no si efectúa, clasificado como comunicación torcida la razón del comunicativa no se extrae del diario de los práxis “del mundo de la vida".

Para Habermas (1998) la sociedad y la cultura son las esferas que si estructuralize confinado por símbolos y, por lo tanto las interpretaciones de la demanda, cualquier método que rechace estas interpretaciones para las cuales el acto social suceda son predestinado a la destrucción. Se entiende, por lo tanto que el ciudadano de la acción del comunicativa es el 
remitente de recepción y/o, ambos activos, configurados en esta perspectiva como producir el agente de la experiencia social de la interacción de la lengua y para el trabajo de los "actos de la opinión” o la "situación ideal del diálogo". Así concluimos que actúa el ciudadano mientras que el agente de su conocimiento apropiado construye significados que determina directo sensible sus traducciones apropiadas de las representaciones de la realidad que si configure con sus experiencias y las experiencias.

El actuar de Comunicativo de Habermas (1998) va de satisfacer a las prerrogativas de la racionalidad instrumental que reduce a ciudadano del conocimiento, suprime la teoría del homogeneiza del conocimiento el proceso de la construcción del conocimiento en los principios de los objetivantes y formal delimitando lo que se debe considerar el conocimiento y los criterios de la verdad, éstos es algunos de los aspectos donde la razón instrumental actúa y consolida el não-concretiza de la existencia del lócus de las relaciones apoyadas en el acuerdo y compartir. La modalidad de la educación interurbana como si estructura y colabora pedagogicamiente, metodologicamente en su programa del plan de estudios y su concepto de aprender con las intenciones de la razón instrumental y amplía su dominio hegemónico que extingue la posibilidad de consolidación de interactivo, comunicativa, totalizante una racionalidad, más extenso, apoyada en la lengua, en el contextualizasión del dialógica al emitir/que recibe si encuentran sumergido en una pelea. El modelo de la relación de el cual los "actos hablan" establece invalida la idea del aislamiento donde si encuentra el cognoscente del ciudadano y el objeto, haciendo posible un horizonte de probabilidad de una comunicación del intersubjetiva que amplía la acción del comunicativa para más allá de los aspectos instrumentales que incluyen así campos de la actividad del universo socio-antroposocio-cultural.

Para insertar la racionalidad del comunicativa en la educación en los medios de la distancia de construir un movimiento del pensante y del estruturante de las nuevas perspectivas capaces para manejar métodos y procedimientos didácticos conduzca por una razón sensible que si distancie de utilitario, industrial, tecnicista una perspectiva compatible con los viejos modelos económicos que vigorizaron la época de su brote que consideraron en la educación en la distancia una solución al triunfo el desafío de la cantidad y ampliar ofertas de ocasiones educativas en niveles distinguidos.

La teoría de la acción del comunicativa puede servir de la inspiración para las transformaciones que son urgentes en la educación en la distancia principalmente en respeto a continuación al redescrever el ciudadano de las posibilidades de interacciones que vayan más 
allá de la transmisión del massiva del contenido que conflui a llegar la dimensión del comunicativa de la lengua. El concepto de la racionalidad diferenció para Habermas (1998) se centra de la manera como adquirimos y utilizamos el conocimiento prendido, al hacer uso el conocimiento de la forma del dialogica que tiene como objetivo a la comprensión, a la interacción de las demandas y, sobre todos los mecanismos para coordinar las acciones que si materialice en a la divergencia, al conflicto y a la tensión de las opiniones.

Sin embargo, la razón del comunicativa en Habermas (1998) sin embargo es una posibilidad no está bastante en mismo sí mismo para la comprensión/la explicación ni la interacción, él debe asignar para articularla un sistema de más grande y un conocimiento más profundo, en la busca de esta dirección para crear una integración, un empalme con como el truncamiento del exploratorio que respetan al sistema de ideas dicen/los conceptos elaboraron para Morin (2002), donde las ojeadas un horizonte de las posibilidades significativas de la construcción de una interacción ética-sociocultural que busca la comprensión de las "circunstancias" que implica cada ciudadano/objeto del proceso.

El pensamiento de Morin construye una síntesis abierta que reencontro de la busca entre la ciencia y el humanismo, cultive científico y la cultura humanistica, que buscar para acumular de la acción el conocimiento complejo con algunas categorías que nominan de bioantropo-sociocultural, refrán mejor, construir conocimiento está antes de cualquier caso que entiende los intermediaciones que si establezca por medio de la cultura, de la sociedad, de la educación, donde el ciudadano necesario aprender ser independiente cuando es desvelar la dirección a aprender vivir y tener el conocimiento de las complejidades de su existencia apropiada.

El humano de la dimensión el que si se relaciona en sus escrituras se relaciona con la afectividad y la subjetividad que adentro las enseña a saber el otro mejor y que, por lo tanto es el conocimiento no sólo en ciencias, pero en el poema que en el profundo madriguera en el alma, del romance que construye un personaje que se despierte adentro en las sensaciones interiores en la relación con otro.

Este ejercicio del humanisation debe consistir de una acción constante vuelta a tomar de de practica mientras que elemento de la formación. En esta dirección, la contribución de tiene como base los conceptos en el profesor reflexivo (Shön, 2000), el uso del monumento como práctico de la automóvil-formación en un curso en la distancia, permite, entre otras las cosas, para entender, como el profesor/la pupila en la distancia construye en la autonomía y la soledad del estudio característico de la modalidad su individualidad apropiada e identidad personal y profesional. 
A través del monumento, el profesor/la pupila es al mismo tiempo sujetos y se opone, un espacio/un momento, donde si él observa, él analiza su práctico y si sea el reencontra en cuanto a (Ferroti), un uno práctico que él considera la vida como espacio de la formación. Segundo Dominicé (en la LECHE, 2005, s/p) “que la historia de la vida pasa para la familia. Es marcado por la escuela. Se orienta para una formación profesional, y en consecuencia beneficia de épocas de la formación continua. La educación se hace así de momentos que adquieran solamente su dirección en la historia de una vida" el profesor social como tema, enreda en un teia de expectativas y de representaciones, que es parte social de una tela, cultural e histórico y que influencie su forma para pensar y para actuar.

En curso de construcción de su identidad, el monumento se convierte en un registro, una narrativa personal en sus actos apropiados, el dar nuevo un significado su profesional práctico, volviendo a tomar la rienda de su desarrollo y de la cuenta para analizar este estruturante social de la tela. En la medida donde él presenta sus impresiones en docto y su correlación con el profesional diario, la pupila/el profesor con la acción-reflexión-acción quita de la experiencia de su apropiado de los lechos de la vida para una acción constante del reformularization de la identidad del profesor y su saber.

Como en un ejercicio constante y solitario, de automóvil-análisis, el monumento permite que entienda las "circunstancias" donde construye su identidad y que evalúe su participación en el proceso de aprendizaje y en el diario del profesor practica. Creemos que los hombres hacen su historia apropiada, dado condiciones. Ampliando este concepto, Heller observa que "los hombres inhalan los ciertos extremos, pero éstos son determinados por "las circunstancias", que, de la porción restante, modifican tales esfuerzos y aspiraciones, produciendo de este modo resultado que el divergem de los extremos deseó inicialmente." (Heller, 2000; p. 01).

Las ofertas de 'las circunstancias' para Heller, de quien los hombres formulan sus propósitos, son relaciones y mediatizadas de los seres humanos de las situaciones para las cosas, no son los paradigmas cerrados, estructuras determinativas del humano de la acción. "Es la unidad de fuerzas productivas, estructura social y formas de pensamiento, es decir, un complejo que contenga posiciones teleologic innumerables, el resultado objetivo de tales posiciones teleologic”.

En esta dirección la historia es la sustancia de la sociedad y los seres humanos de las acciones, cuando los publicizadas, adquieren un peso más grande en el contexto social, un "papel social" en el contexto de la vida de cada día. Como los resultados sociales de la 
función de algunos factores de la vida de cada día, factores ilimitados, él "producto en el hombre la necesidad para modificarse permanentemente, para renovarse, a cambiado. Esta necesidad de la novedad, la necesidad para transformar constantemente a la sociedad de tal manera cuánto ourselves, somos una de las conquistas más grandes del humano de la historia”. (Heller, de Op. Sys. CIT; 91)

\section{El final de Considérasion.}

Que toma como base los conceptos en el profesor reflexivo (Shön, 1998), el uso del monumento como práctico de la automóvil-formación en un curso en la distancia, permite, entre otros las cosas, para entender, como el profesor/la pupila en la distancia construye en la autonomía y la soledad del estudio característico de la modalidad su individualidad apropiada e identidad personal y profesional.

En curso de construcción de su identidad, el monumento se convierte en un registro, una narrativa personal en sus actos apropiados, el dar nuevo un significado su profesional práctico, volviendo a tomar la rienda de su desarrollo y de la cuenta para analizar este estruturante social de la tela. En la medida donde él presenta sus impresiones en docto y su correlación con el profesional diario, la pupila/el profesor con la acción-reflexión-acción quita de la experiencia de su apropiado de los lechos de la vida para una acción constante del reformularization de la identidad del profesor y su saber.

Para entender de cuando sea sensible las memorias y las narrativas del monumento hacen posible un ejercicio permanente de la construcción/de la reconstrucción practican a profesor, establecido en la reflexión como mediación de la acción, asistiendo a los educadores en la aprehensión y la transformación apropiada de el práctico.

El registro de experiencias y usted practican, son marcas de la reflexión constitutiva de la formación de enseñanza, por lo tanto van profundamente a saber y la opinión a ellas de los profesores como reflexivos profesionales, de los investigadores y en de sí mismo y de su acción que construyen nosotros de una red de referir a los teóricos que apoyan la practica pedagógica, constituido y del constituidora de una para saber para hacer para la experiencia o qué Josso, define como "experiencia del formadora", "aprender experiencial" y "memoriareferencias" (Josso, 2000). 
Uno de los resultados identificados en el uso del monumento como proceso de la construcción de la identidad del profesor/de la pupila habita en la actual perspectiva de liberación en el ejercicio continúa y reflexivo de la lengua, (en este caso de la lengua escrita). En el acto del registro de su acción, reacciones, avances y retrocesos, de la forma crítica y continua los avances de la pupila/del profesor del habitus simple de la identificación, establecieron mucho más en "doxa", para el campo de la identidad concreta mientras que proceso de la apropiación e incorporación de las acciones prácticas y en la formación del profesional de la educación. En la perspectiva del freiriana el lanzamiento del ciudadano esto en el proceso crítico de la identificación de esto en el mundo, en un ejercicio de pertenecer substantivo y actual en el par de la sierra que va conforme al mundo y de éste de o al ciudadano.

El monumento, como ejercicio de la reflexión en practica para aprender y enseñar contribuye para vuelta a tomar de reflexiones en practica el diario pedagógico para enseñar y para aprender, haciendo con eso la poder del profesional de la educación interpreta y a su reinterpretar practica el percibir de él como política de la acción y, por lo tanto la relación de ser ese capaz necesita ser; critica, planeado y creativo.

\section{Referencias bibliográficas.}

CUNHA, Maria Antonieta Antunes. Guia Geral do PROFORMAÇÃO, $1^{\mathrm{a}}$ e $3^{\mathrm{a}}$. Ed. Brasília: MEC. FUNDESCOLA, 2000.

D’AMBRÓSIO U. Tempo da escola e tempo da sociedade. In SERBINOO, Raquel V. RIBEIRO, Ricardo. BARBOSA, Raquel L. Leite.e GEBRAN, Raimunda A. (org.) Formação de Professores. São Paulo, UNESP, 1998, p. 242.

HABERMAS, J. Teoria de La Acción Comunicativa. Racionalidad de La Acción y Racionalización Social. Madrid, Taurus. Tomo I, II. 1988.

HALL, Stuart. A identidade cultural na pós-modernidade/. 3. ed. Rio de Janeiro: DP\&A, 1999.

Stuart. Identidade cultural e diáspora In. /Revista do Patrimônio/, $\mathrm{n}^{\mathrm{o}} 24$, Instituto do Patrimônio Histórico e Artístico Nacional do Ministério da Cultura, Brasília, 1996. 
Stuart. A centralidade da cultura: notas sobre as revoluções culturais do nosso tempo. In. /Revista Educação e Realidade/. Porto Alegre, Faculdade de Educação /UFRGS. vol. 22/nº 02. Jul/dez,1997.

HELLER, A. /O cotidiano e a história/. 6. ed. São Paulo: Paz e Terra. 2000.

JOSSO, Marie-Christine. Experiências de vida e formação. São Paulo: Cortez, 2004.

LEITE, Laizza Carvalho Santos. Memórias de alfabetização das professoras do Sertão Baiano In www.cereja.org.br/arquivos_upload/laizza\%2520carvaho_s_leite_visemana_2005.pdf , capturado em 05/07/2006.

LÜDKE, M. (coord.). /O professor e a pesquisa. /Campinas, SP: Papirus, 2001.

, M. A complexa relação entre o professor e a pesquisa. In: ANDRÉ, M. (org.). /O papel da pesquisa na formação e na prática dos professores. /Campinas, SP: Papirus, 2001.

M. A pesquisa em educação: conceitos, políticas e práticas. In: GERALDI, Corinta M. e outros. Cartografias do Trabalho docente Campinas/São Paulo; Mercado de Letras, 1998. Não está citado ao longo do texto.

MATURANA, H. A ontologia da realidade. Belo Horizonte: Editora UFMG.1997. \& VARELA, F. A árvore do conhecimento. Campinas: Editora Psy, 1989.

MORIN, E. Ciência com consciência. Trad.: Maria D.Alexandre e Maria Alice Sampaio Dória. 2a ed. Rio de Janeiro: Bertrand Brasil, 1998.

MORIN, Edgar. O Método IV: As Idéias, Habitat, Vida, Costumes, Organização. Trad. Juremir Machado da Silva. 3ª Ed. Porto Alegre: Sulina, 2002.

MORIN, E. \& LÊMOIGNE, J. L. A Inteligência da complexidade. São Paulo: Fundação Peirópolis, 2000, p. 220

PÉREZ GÓMES, Angel I. O Pensamento prático do professor: a formação do professor como profissional reflexivo. In: NOVOA, Antonio. Os Professores e sua formação. Lisboa: Publicação D. Quixote, 1995. 
NÓVOA, Antonio. /Os professores e a sua formação/ (coord.). Lisboa/Portugal: Publicações Dom Quixote, 1992. (Temas de Educação 1) . /Profissão professor./

Porto/Portugal: Porto, 1995. (Coleção Ciências da educação).

SHÖN, D. Educando o profissional reflexivo: um novo design para o ensino e a aprendizagem. /Porto Alegre, RS: ArtMed, 2000a.

Formar professores como profissionais reflexivos. In: NÓVOA, A. /Os professores e sua formação. /Lisboa, Portugal: Dom Quixote, 1992. 